\title{
Structure function analysis and intermittency in the atmospheric boundary layer
}

\author{
J. M. Vindel ${ }^{1}$, C. Yagüie $^{2}$, and J. M. Redondo ${ }^{3}$ \\ ${ }^{1}$ Agencia Estatal de Meteorología, Madrid, Spain \\ ${ }^{2}$ Dept. Geofísica y Meteorología, Universidad Complutense de Madrid, Spain \\ ${ }^{3}$ Dept. Física Aplicada, Universidad Politécnica de Cataluña, Barcelona, Spain \\ Received: 10 April 2008 - Revised: 31 July 2008 - Accepted: 1 October 2008 - Published: 27 November 2008
}

\begin{abstract}
Data from the SABLES98 experimental campaign (Cuxart et al., 2000) have been used in order to study the relationship of the probability distribution of velocity increments (PDFs) to the scale and the degree of stability. This connection is demonstrated by means of the velocity structure functions and the PDFs of the velocity increments.

Using the hypothesis of local similarity, so that the third order structure function scaling exponent is one, the inertial range in the Kolmogorov sense has been identified for different conditions, obtaining the velocity structure function scaling exponents for several orders. The degree of intermittency in the energy cascade is measured through these exponents and compared with the forcing intermittency revealed through the evolution of flatness with scale.

The role of non-homogeneity in the turbulence structure is further analysed using Extended Self Similarity (ESS). A criterion to identify the inertial range and to show the scale independence of the relative exponents is described. Finally, using least-squares fits, the values of some parameters have been obtained which are able to characterize intermittency according to different models.
\end{abstract}

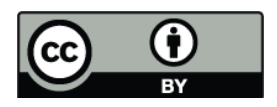

Correspondence to: J. M. Vindel (jmvindel@inm.es)

\section{Introduction}

The single point velocity structure function of order $p$, for a certain temporal scale $(\tau)$, is defined as:

$S_{p}(\tau)=\left\langle|u(t+\tau)-u(t)|^{p}\right\rangle$

Richardson (1922) assumed the existence of an energy cascade, which was formalized along the so-called inertial range by Kolmogorov $(1941,1962)$. The spatial and temporal descriptions of the cascade may be exchanged assuming Taylor's "frozen eddy" hypothesis (Stull, 1988), but care is needed in non-homogeneous flows (Mahjoub et al., 1998). Within the inertial range, for homogeneous and isotropic turbulence in local equilibrium, Kolmogorov (1941) established the following relation between the spatial structure functions and length scales $(l)$ :

$S_{p}(l) \sim\langle\varepsilon\rangle^{p / 3} l^{p / 3}$

where $\langle\varepsilon\rangle$ is the mean energy dissipation rate assuming that it does not vary in space or time. This inertial range spreads out from the integral scale (of the order of the scale which generates the perturbation) to the Taylor microscale (of the three standard turbulence length scales, the one for which viscous dissipation begins to affect the eddies) and continues further to smaller scales defined by Kolmogorov (the scale that characterizes the smallest dissipation-scale eddies; Glickman, 2000).

Whether we consider spatial or time dependence, if the conditions of existence of an equilibrium inertial range are satisfied, a potential type relationship is observed between the structure functions or different order, $p$, and the scale (e.g. Anselmet et al., 1984, for shear flows):

$S_{p}(\tau) \sim \tau^{\zeta p}$

Published by Copernicus Publications on behalf of the European Geosciences Union and the American Geophysical Union. 


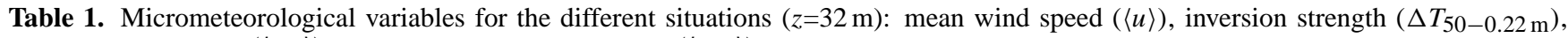
wind speed variance $\left(\left\langle u^{\prime 2}\right\rangle\right)$, vertical wind speed variance $\left(\left\langle w^{\prime 2}\right\rangle\right)$, gradient Richardson number $(R i)$, Kolmogorov scale $\left(\tau_{k}\right)$, Taylor scale $\left(\lambda_{\tau}\right)$ and time integral scale $\left(T_{0}\right)$.

\begin{tabular}{lrrrrrr}
\hline Day_time(GMT) & $14 \_00: 00$ & $14 \_06: 00$ & $14 \_12: 00$ & $14 \_18: 00$ & $15 \_00: 00$ & $15 \_06: 00$ \\
\hline$\langle u\rangle\left(\mathrm{m} \mathrm{s}^{-1}\right)$ & 7.65 & 6.32 & 8.42 & 7.69 & 3.05 & 3.75 \\
$\Delta \mathrm{T}_{50-0.22 \mathrm{~m}}(\mathrm{~K})$ & 0.65 & 1.63 & -3.81 & 0.01 & 5.11 & 5.99 \\
$\left\langle u^{\prime 2}\right\rangle\left(\mathrm{m}^{2} \mathrm{~s}^{-2}\right)$ & 0.2558 & 0.288 & 2.7226 & 0.4603 & 0.0559 & 0.0134 \\
$\left\langle w^{\prime 2}\right\rangle\left(\mathrm{m}^{2} \mathrm{~s}^{-2}\right)$ & 0.1002 & 0.0794 & 0.6724 & 0.14 & 0.006 & 0.002 \\
$R i$ & -0.0001 & 0.0011 & -0.3851 & 0.0355 & 0.0908 & 0.1967 \\
$\tau_{k}(\mathrm{~s} / 20)$ & 0.000516 & 0.00055 & 0.000637 & 0.000497 & 0.003361 & 0.002197 \\
$\lambda_{\tau}(\mathrm{s} / 20)$ & 0.16770 & 0.13031 & 0.30969 & 0.15690 & 1.13049 & 0.73006 \\
$T_{0}(\mathrm{~s} / 20)$ & 152.39 & 62.71 & 629.56 & 134.76 & 1100.75 & 694.21 \\
\hline
\end{tabular}

where $\zeta_{p}$ is the so-called scaling exponent, now defined in time, but with the same value as the spatial ones, providing Taylor's hypothesis holds (Stull, 1988). Willis and Deardorff (1976) showed that in order to satisfy the requirement for the eddy to have negligible change as it advects past a sensor, the wind standard deviation should be less than half the value of wind speed, and this condition is fulfilled by our data.

According to Kolmogorov's (1941) initial theory, the mean energy dissipation rate, $\langle\varepsilon\rangle$, along the different scales of the Richardson cascade was expected to remain constant. Kolmogorov established a linear dependence of the exponents $\zeta_{p}$ as a function of the order $p$. However, experimentally, first Batchelor and Townsend (1949), and then Landau (see Landau and Lifshitz, 1959; Frisch, 1995), observed deviations from Kolmogorov's theory. This phenomenon is generally known as intermittency of the turbulence cascade, and is characterized by the anomalous scaling exponents (deviations from the exponents $\zeta_{p}=\mathrm{p} / 3$ foreseen by Kolmogorov, 1941). In a more refined theory, assuming a lognormal variation, Kolmogorov (1962) introduced the possibility of scale dependence of dissipation, which sparked a wealth of different phenomenological models of turbulence, (see Frisch, 1995, for an historical account) that give several approximations to these exponents. Other methods try to derive intermittency directly from Navier-Stokes equations (see, e.g., Grossmann et al., 1994; Giles, 2001; or more recently, Angheluta et al., 2006, for the case of a nonlinear model of turbulence).

The local dissipation $\langle\varepsilon\rangle=\left\langle v \omega_{i j} \omega_{j i}\right\rangle$, with $v$ the kinematic viscosity and $\omega$ the vorticity so that $\omega_{i j}=\left(\partial u_{i} / \partial x_{j}-\partial u_{j} / \partial x_{i}\right)$ is generally a very complicated (multifractal) function. When the local energy dissipation is not constant with the scale, it is said that intermittency is present, but we should note that we may be including other effects, such as non-homogeneity or anisotropy, within a single concept. The probability distribution of $\varepsilon$ (or of other variables dependent on the scale, as is the case with the velocity or temperature increments or their derivatives) will differ according to the scale taken into account. In the range between the integral scale, $L_{0}$, (the scale of the order of disturbance generated by the turbulence) and the Taylor microscale, (where production and dissipation are in equilibrium) which we will call macroturbulence (Rodriguez et al., 1999), the corresponding normalized PDF usually appears Gaussian and, as a result of intermittency, modifies its shape more and more as the scale diminishes (see, e.g. Biferale, 1993; Li and Meneveau, 2005; or Li and Meneveau, 2006).

Thus, an index that characterizes the variation of probability distribution could be introduced as an intermittency index (of macroturbulence) related to flatness or Kurtosis. For example, Chevillard et al. (2005), considered the direct measure of $\log \left(\frac{F}{3}\right)$, where $F$ is the flatness of the velocity. Bottcher et al. (2007) have recently shown an example of the evolution of flatness with scale: for large scales, the flatness has values around 3 (Gaussian flatness), and it increases as the PDF appears more and more pointed. Another method of characterizing the intermittency of the cascade in the Kolmogorov (1962) sense is to measure the difference between 2 and the sixth order scaling exponent of the structure function, $\mu=2-\zeta_{6}$, but we should observe that this simply includes in a single parameter the many possible deviations from the Kolmogorov (1941) result $\left(\zeta_{p}=\mathrm{p} / 3\right)$, including non-homogeneity and non-locality.

In this article, both indices of intermittency (scaling exponents and variation of flatness) will be measured for atmospheric boundary layer velocity data and related and, 

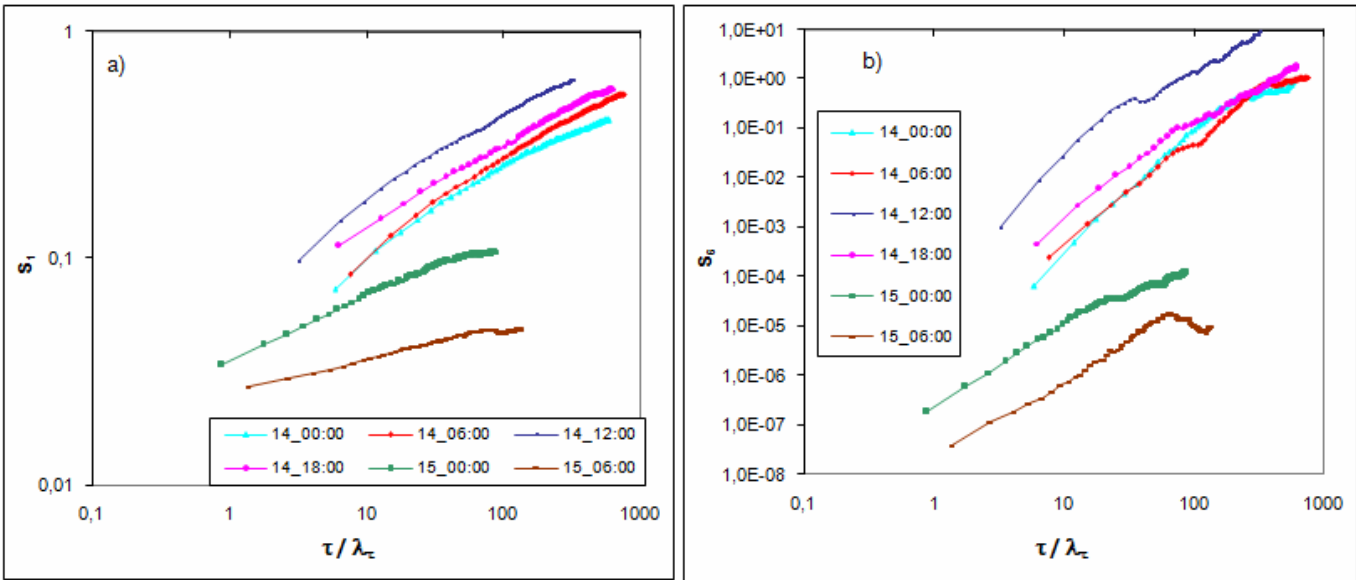

Fig. 1. Evolution of the structure functions with the scale for different stratification situations. (a) order 1; (b) order 6.

although with the present data it is not possible to establish a univocal correlation between the type of stratification and the degree of existing intermittency, a stronger dependence is found between the type of stratification and the structure functions. First we present the data, followed by the PDF and structure functions analysis, a statistical convergence study, the flatness and scaling exponents results, and finally the application of Extended Self Similarity and some other intermittent scaling theories.

\section{Data}

SABLES98 (Stable Atmospheric Boundary Layer Experiment in Spain 1998) data (Cuxart et al., 2000) from a sonic anemometer $(20 \mathrm{~Hz}$ sampling rate) at $32 \mathrm{~m}$ were used. This field campaign took place in September 1998 (from 10 to 28) at the Research Centre for the Lower Atmosphere (CIBA) which is situated on the Northern Spanish Plateau. The surrounding terrain is fairly flat and homogeneous. In the experiment, different degrees of stable stratification were achieved during the night, from near-neutral to very stable conditions.

The analysis presented here was done for six different stability situations (with a temporal lag of $6 \mathrm{~h}$ ) between 14 September at 00:00 GMT and 15 September at 06:00 GMT. Accordingly, this work examines not only nocturnal data, but also diurnal situations where convective instability was present. Turbulent and stability parameters as well as mean wind speed can be found in Table 1, although further information can be found in Yagüe et al. (2006).

A bulk index of the existing stability degree in each situation is the difference of temperature between the levels of 50 and $0.22 \mathrm{~m}$ showing the strength of surface-based inversion developed during the night, or the convective lapse rate during the day. It is also important to measure the level of stratification by calculating the local gradient Richardson number. This non-dimensional number takes into account the local vertical wind shear as well as the density gradient. In the turbulent kinetic energy equation, the Flux Richardson number is the ratio of the buoyancy and production terms, and relating fluxes to gradients (Redondo et al., 1996) it is possible to measure $R i$ locally in general as:

$R i=g \Delta \rho L_{0} / \rho u^{\prime 2}$

where $\Delta \rho$ is the density difference and $L_{0}$ is the integral scale. From the actual measured temperature and horizontal velocity data, the gradient Richardson number is defined (Cuxart et al., 2000) as:

$R i=\frac{\frac{g}{\theta_{0}} \frac{\partial \bar{\theta}}{\partial z}}{\left(\frac{\partial \bar{u}}{\partial z}\right)^{2}+\bar{u}^{2}\left(\frac{\partial \bar{\alpha}}{\partial z}\right)^{2}}$

To evaluate the gradients of wind velocity $(u)$ and potential temperature $(\theta), \log$-linear fits (Nieuwstadt, 1984) were made to the different levels data. The wind direction $(\alpha)$ gradient is evaluated using simple linear fits:

$\bar{u}=a z+b \ln z+c$
$\bar{\theta}=a^{\prime} z+b^{\prime} \ln z+c^{\prime}$
$\bar{\alpha}=a^{\prime \prime} z+c^{\prime \prime}$

It may be established from Table 1 (where all the stability and turbulent parameters agree) that on 14 September at 12:00 GMT there was a clearly convective instability situation, and that on 15 September at 06:00 GMT and at 00:00 GMT there occurred the most stable situations (higher inversion strengths and Richardson numbers, lower wind speeds and horizontal and vertical variances). However, smaller and less clear differences are found on 14 September at 00:00 GMT, 06:00 GMT and 18:00 GMT: according to the Richardson number, 00:00 GMT is the most neutral situation, while 06:00 GMT is the situation where vertical turbulent transfer is most inhibited (lower vertical covariance, lower wind speed and greater inversion strength). 

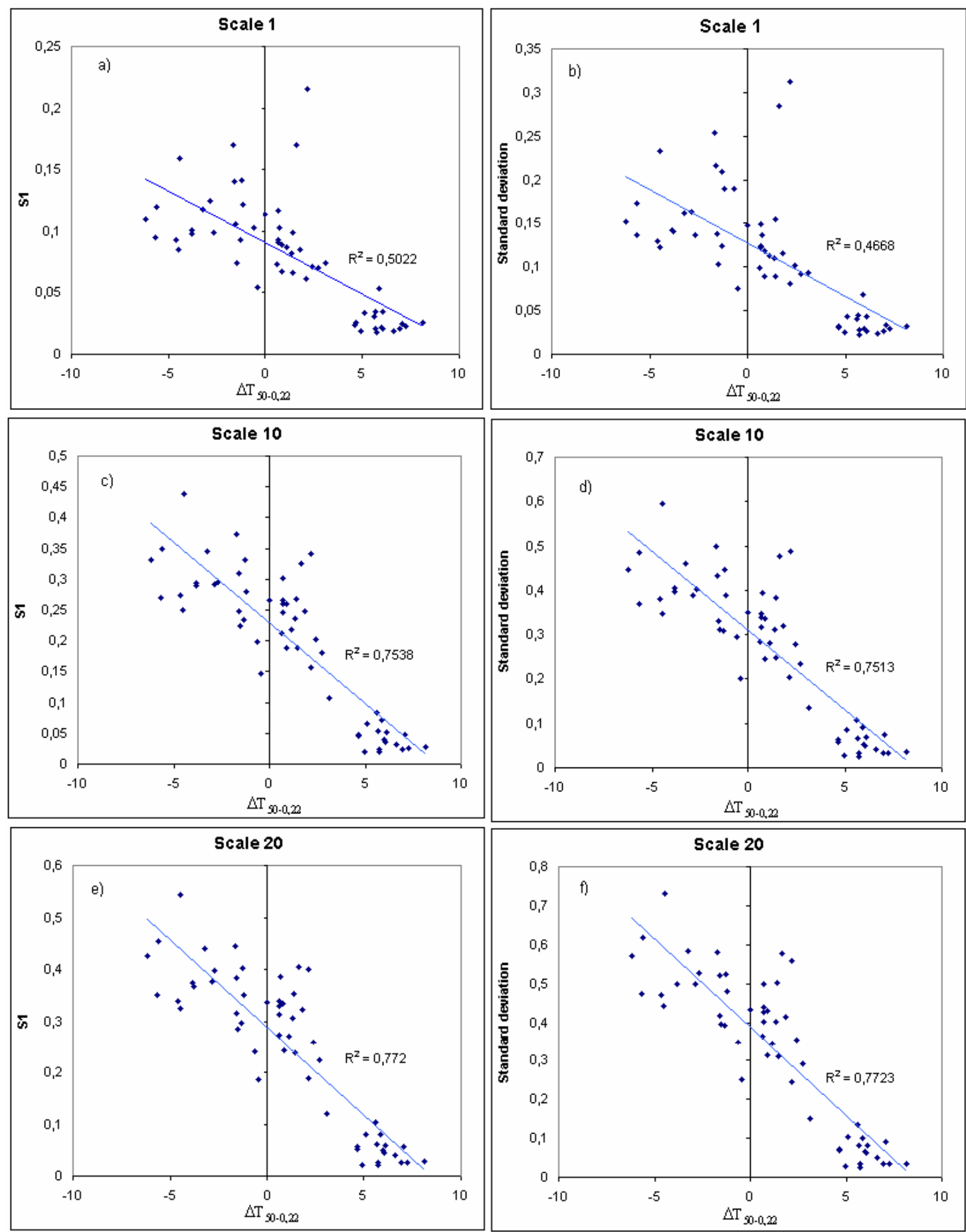

Fig. 2. Order 1 structure function (left) and Standard deviation (right) versus $\Delta T_{50-0.22 \mathrm{~m}}$ for a certain scale: (a) and (b): 1 (s/20); (c) and (d): 10 (s/20); (e) and (f): 20 (s/20) (Dots represent conditions every 30 minutes) (Including the coefficient of determination corresponding to least-squares linear fit). 

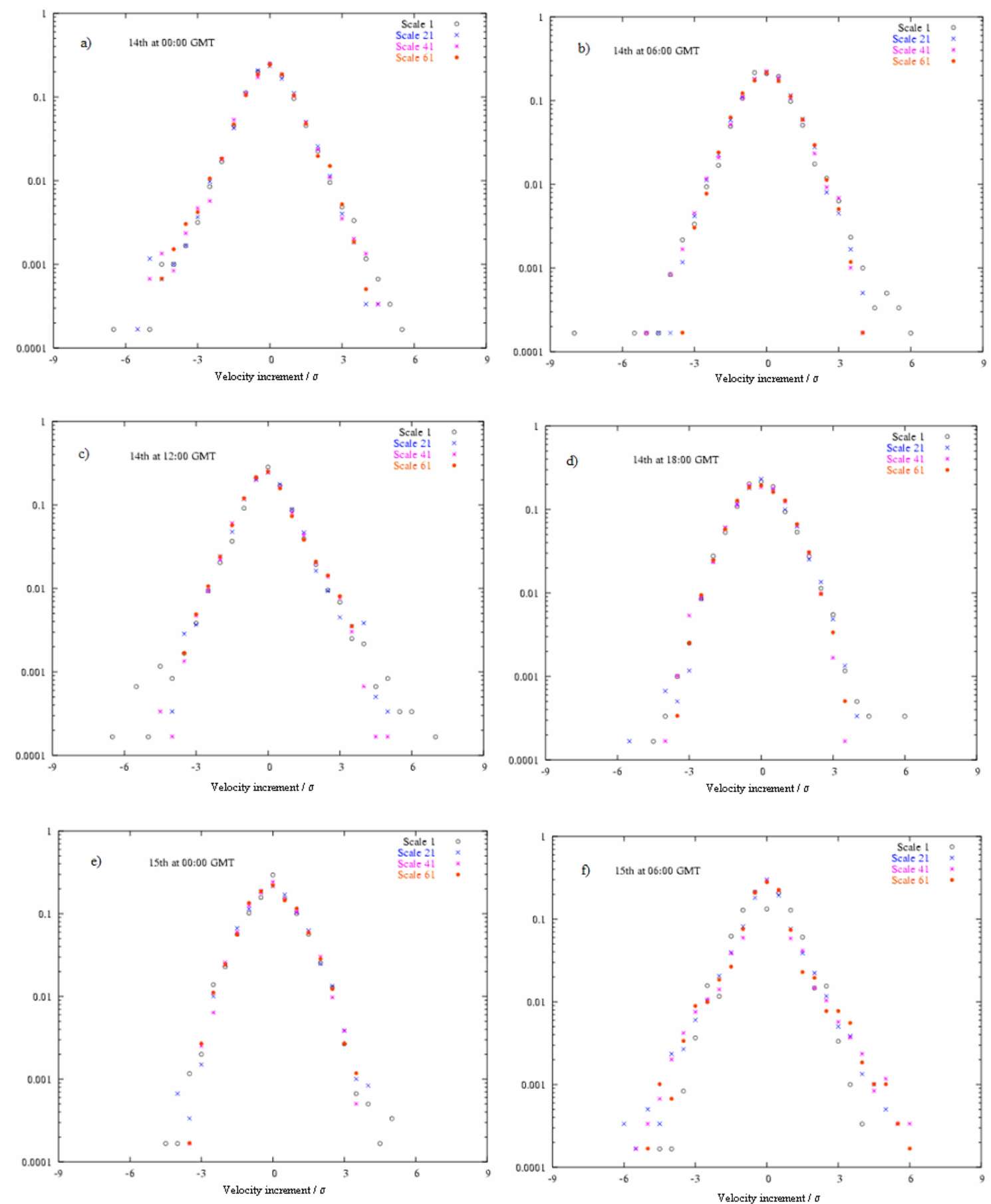

Fig. 3. PDFs of velocity increments for different scales: (a) the 14th at 00:00 GMT; (b) the 14th at 06:00 GMT; (c) the 14th at 12:00 GMT; (d) the 14th at 18:00 GMT; (e) the 15th at 00:00 GMT; (f) the 15th at 06:00 GMT. 
Table 2. Estimated scale ranges (s/20) for the different situations analyzed.

\begin{tabular}{lllllll}
\hline Day_Time (GMT) & $14 \_00: 00$ & $14 \_06: 00$ & $14 \_12: 00$ & $14 \_18: 00$ & $15 \_00: 00$ & $15 \_06: 00$ \\
\hline Range (Kolm) & {$[2-59]$} & {$[2-81]$} & {$[1-74]$} & {$[3-14]$} & Not possible & Not possible \\
\hline
\end{tabular}

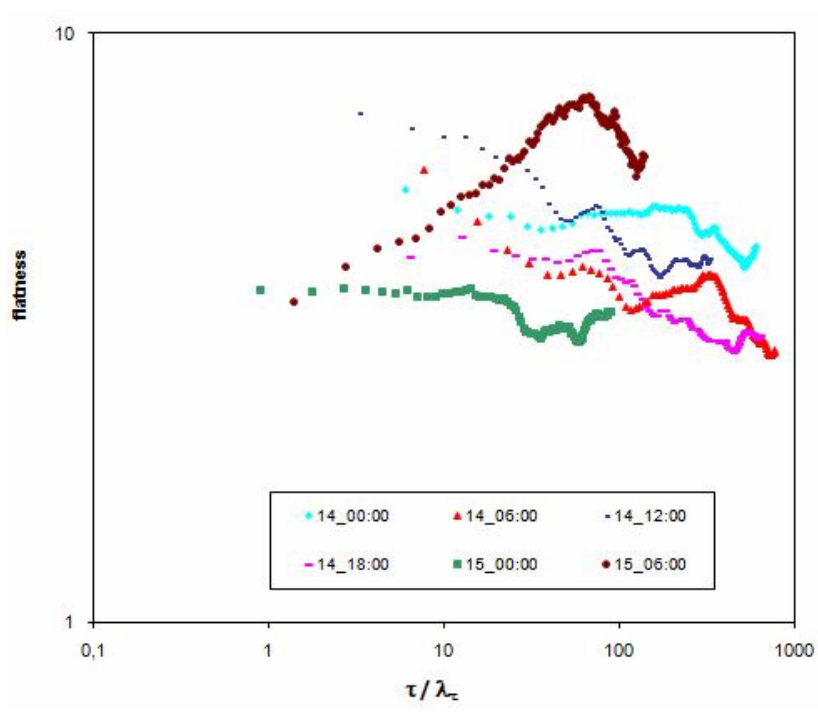

Fig. 4. Evolution of flatness with scale for different stratification situations.

In Table 1 are included the typical Kolmogorov, Taylor and Integral scales, corresponding to the different situations. In the literature, to compare numerical simulations of turbulence, the scales are usually non-dimensionalized by Taylor's scale, and in this work this has been done to compare different stability situations.

The Kolmogorov and Taylor spatial and temporal scales correspond to the following expressions (Tennekes and Lumley, 1994):

$$
\begin{aligned}
& \eta_{k}=A^{(-1 / 4)} R e_{\mathrm{int}}^{(-3 / 4)} L_{0} \equiv\left(\frac{v^{3}}{\varepsilon}\right)^{1 / 4} ; \tau_{k}=\frac{\eta_{k}}{U} \\
& \lambda_{l}=\left(\frac{15}{A}\right)^{(1 / 2)} \operatorname{Re}_{\mathrm{int}}^{(-1 / 2)} L_{0} ; \lambda_{\tau}=\frac{\lambda_{l}}{U}
\end{aligned}
$$

where $A$ is a constant of the order of 1 , which we will suppose equal to 0.5 (Balbastro et al., 2004), $R e_{\text {int }}$ is the Reynolds number corresponding to the integral scale $\left(L_{0}\right)$ and $U$ is the wind speed.

Five-minute series (6000 data points) were used to study each situation, which is an optimal compromise between using enough data to provide the statistics and avoiding mesoscale motion influences, as we are interested in the turbulent scales (Stull, 1988).
In order to investigate the relationship between structure functions and stability, five-minute series were analysed every 30 min between 14 September (00:00 GMT) and 15 September (06:00 GMT). The variable studied was the horizontal velocity component in the mean flow direction $(u)$, thus all further conclusions only apply to the horizontal structure of the boundary layer turbulence. It is clear that for less stratified turbulence the cascade will be more threedimensional, while the reduction of the vertical scales below Ozmidov's length scale as stratification increases will flatten Reynolds' stresses in spite of a possible internal wave field.

\section{Analysis of structure functions and PDFs}

The potential relationship produced by Eq. (3) is shown in Fig. 1, where two structure functions (order 1 and order 6) have been represented for different situations. It is observed that by far the highest values for structure functions correspond to 14 September at 12:00 GMT, followed by the same day at 18:00 GMT and 06:00 GMT; then 14 September at 00:00 GMT and, finally, with values much lower than the previous ones, the situations represented for the 15 th.

Higher values of structure functions imply a higher range for velocity increment values. That is to say, the dispersion of these increments is greater, due to a lack of homogeneity. Structure functions and the degree of stratification must therefore present a certain relationship, as shown in Fig. 2, where an increase in the value of the structure functions may be observed (specifically of $S_{1}$ and standard deviation - square root of $S_{2}-$ ), as the instability is increased (lower values in the temperature difference between the levels of 50 and $0.22 \mathrm{~m}$.).

On the other hand, the phenomenon of intermittency alludes to infrequent events, which correspond to the velocity increments furthest from zero. Thus the greater or lesser stretching of the tails in the PDFs is indicative of the (greater or lesser) grade of intermittency (Frisch, 1995; Sorriso-Valvo et al., 2000; Chevillard et al., 2006). In order to study these behaviour patterns, in Fig. 3 we have represented the PDFs (in log scale) of the velocity increments normalized by the Standard Deviation, corresponding to different scales and for the different situations. The following characteristics are observed:

1. The behaviour pattern of the scales, in a given situation, proves to be quite similar for the larger scales. The most 

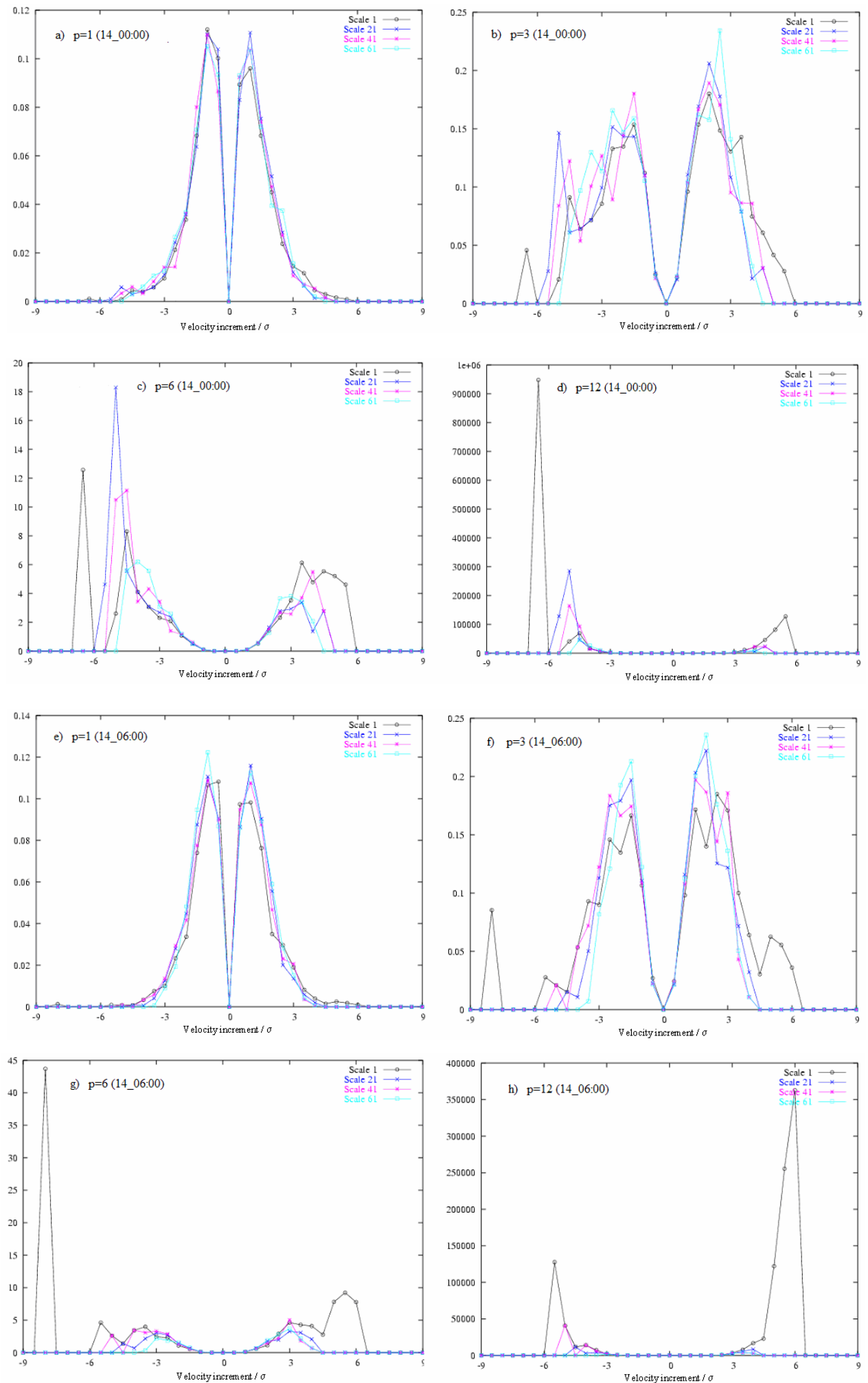

Fig. 5. Product of the $p$-power ( $p=1,3,6$ and 12) of the velocity increments by its corresponding probability versus the velocity increments for different scales: (a), (b), (c), and (d) for the 14th at 00:00 GMT; (e), (f), (g) and (h) for the 14th at 06:00 GMT. 

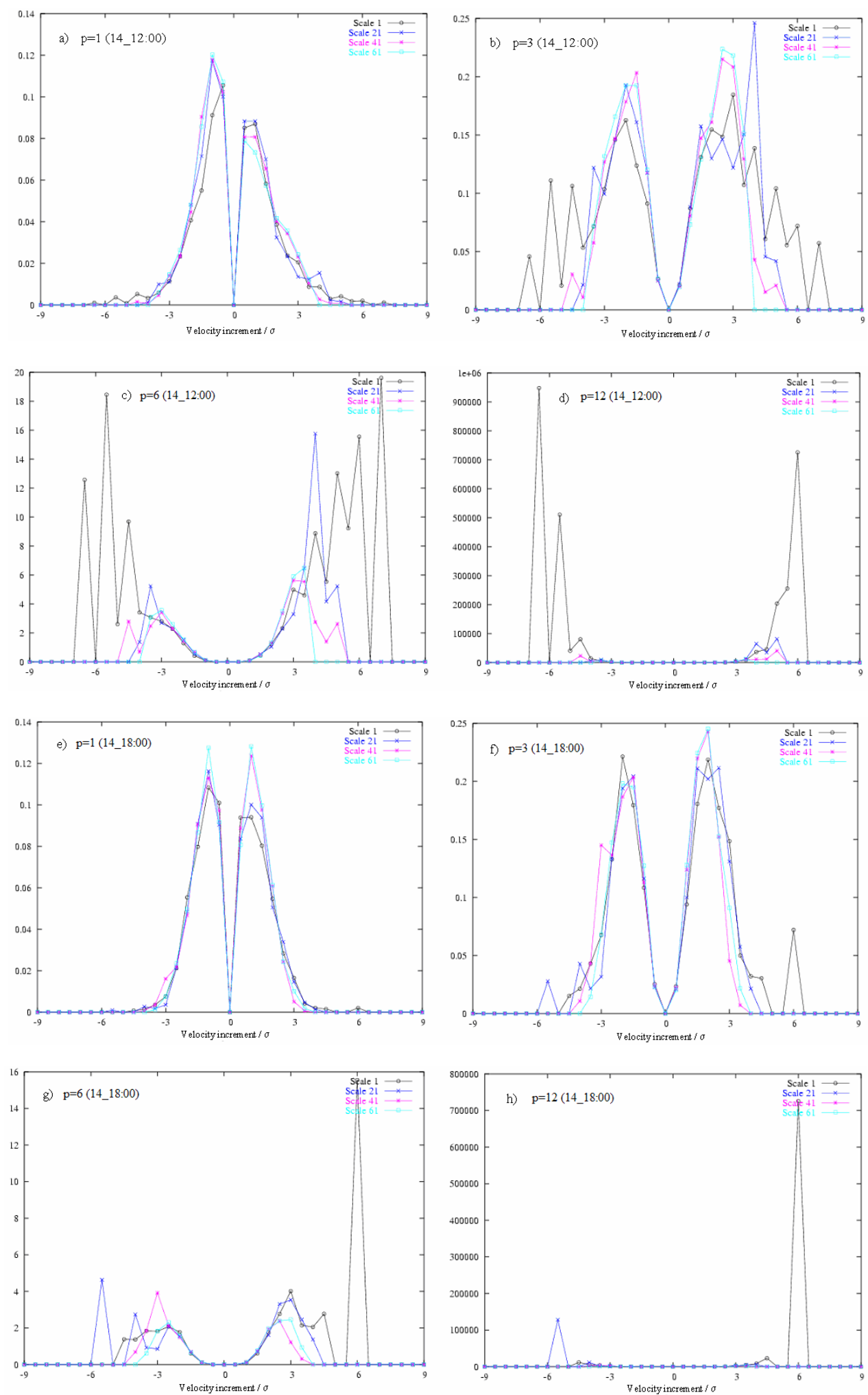

Fig. 6. Product of the $p$-power ( $p=1,3,6$ and 12) of the velocity increments by its corresponding probability versus the velocity increments for different scales: (a), (b), (c), and (d) for the 14th at 12:00 GMT; (e), (f), (g) and (h) for the 14th at 18:00 GMT. 

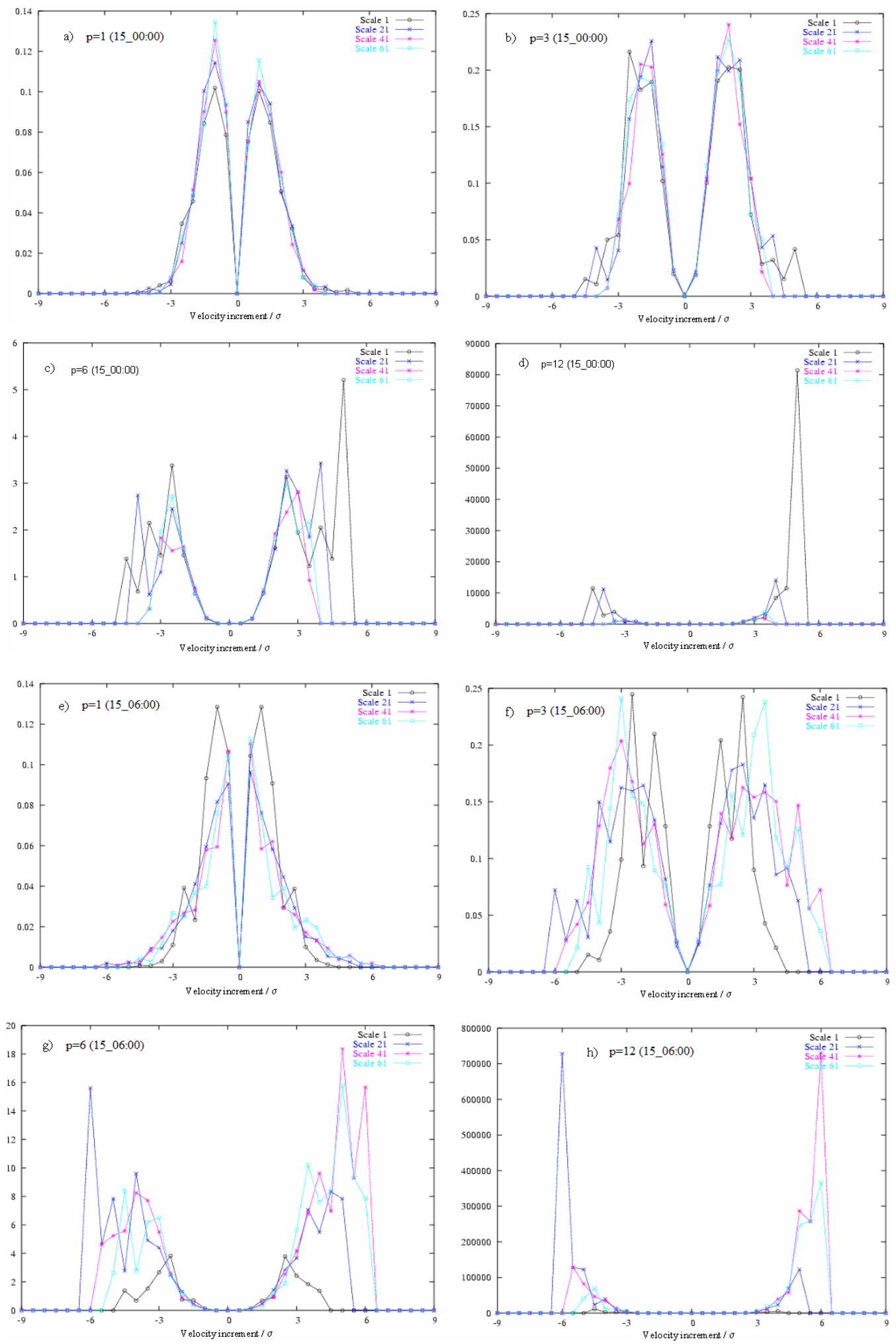

Fig. 7. Product of the $p$-power ( $p=1,3,6$ and 12) of the velocity increments by its corresponding probability versus the velocity increments for different scales: (a), (b), (c), and (d) for the 15th at 00:00 GMT; (e), (f), (g) and (h) for the 15th at 06:00 GMT. 


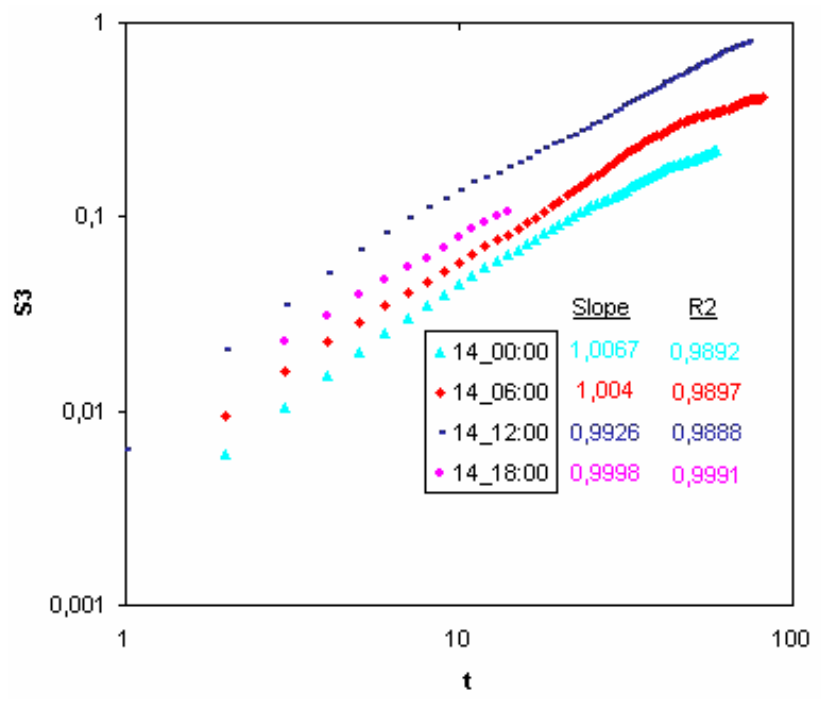

Fig. 8. Slope of least-squares linear fit and coefficients of determination between $\log$ of the order 3 structure function and $\log$ of the scale for different stratification situations.

significant differences are appreciated in the tails of the smaller scales.

2. In Fig. $3 b$ and $c$ the tails of the smaller scales stretch further than in the other cases, indicative of a higher degree of intermittency. On the other hand, in Fig. 3e and $\mathrm{f}$ (the most stable situations) the tails of the smaller scales are more closed, especially in case $3 \mathrm{f}$, where it may be seen that the PDF of scale 1 falls within the other PDFs.

The flatness of the PDFs shown above is statistically defined from the structure functions as:

$F=\frac{S_{4}}{\left(S_{2}\right)^{2}}$

As we have already said, flatness seems to be a very good indicator of the degree of existing intermittency: Biferale et al. (2008) point out that, when flatness changes with scale following a potential law, intermittency is present. Figure 4 shows, in log-log scale, the evolution of flatness with scale. It may be quite clearly seen that the greatest decrease of flatness with scale corresponds to 14 September at 12:00 GMT. However, the increase of flatness for the 15th at 06:00 GMT is particularly significant, in accordance with the closing of the PDFs for the smaller scales as mentioned above.

In order to show the degree of statistical convergence displayed by the structure functions of the successive orders, we calculated for velocity increments, the product of the corresponding probability by the $p$-power of those increments (Schumacher, 2001). The area below the curve thus obtained corresponds to the moment of order $p$ and, consequently, the statistical convergence of that moment will improve when

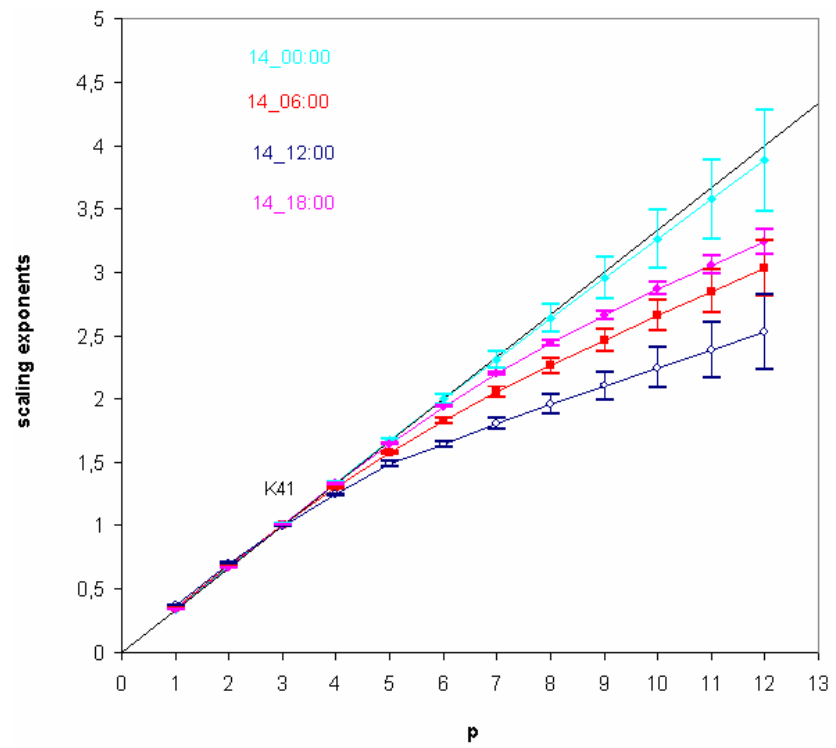

Fig. 9. Scaling exponents versus order of the structure function for different situations.

the scatter of the curve decreases. In Figs. 5, 6 and 7 we have shown the curves mentioned above for orders $1,3,6$ and 12 for the different situations analysed and various different scales.

Certainly, for orders higher than 6 we may already observe a considerable scattering for certain values of the tails, which we consider satisfactory because with meteorological field data we are far from controlled laboratory wind tunnel conditions. The lack of strict statistical convergence for orders higher than 3 does not suppose any limitation to the method of analysis described here. On the other hand it stimulates the analysis of wind data at much higher frequency. Nevertheless, because most of the data scattering and lack of convergence can be seen to take place fundamentally when the scale diminishes, at about the Taylor microscale, we may forward the hypothesis that the absence of convergence might be motivated by the existence of intermittence precisely in the non-equilibrium situations: stratified 2-D type turbulence and convective 3-D unstable situations. From our point of view the overall results of this work are relevant even if we limit the study to the smaller orders, where the convergence is much higher.

Figures 3, 4, 5, 6 and 7 prove to be convergent as to the possible intermittency shown by the different cases, with a very marked intermittency on the 14th at 12:00 GMT and the opposite result on the 15 th, where there is considerable stability. 

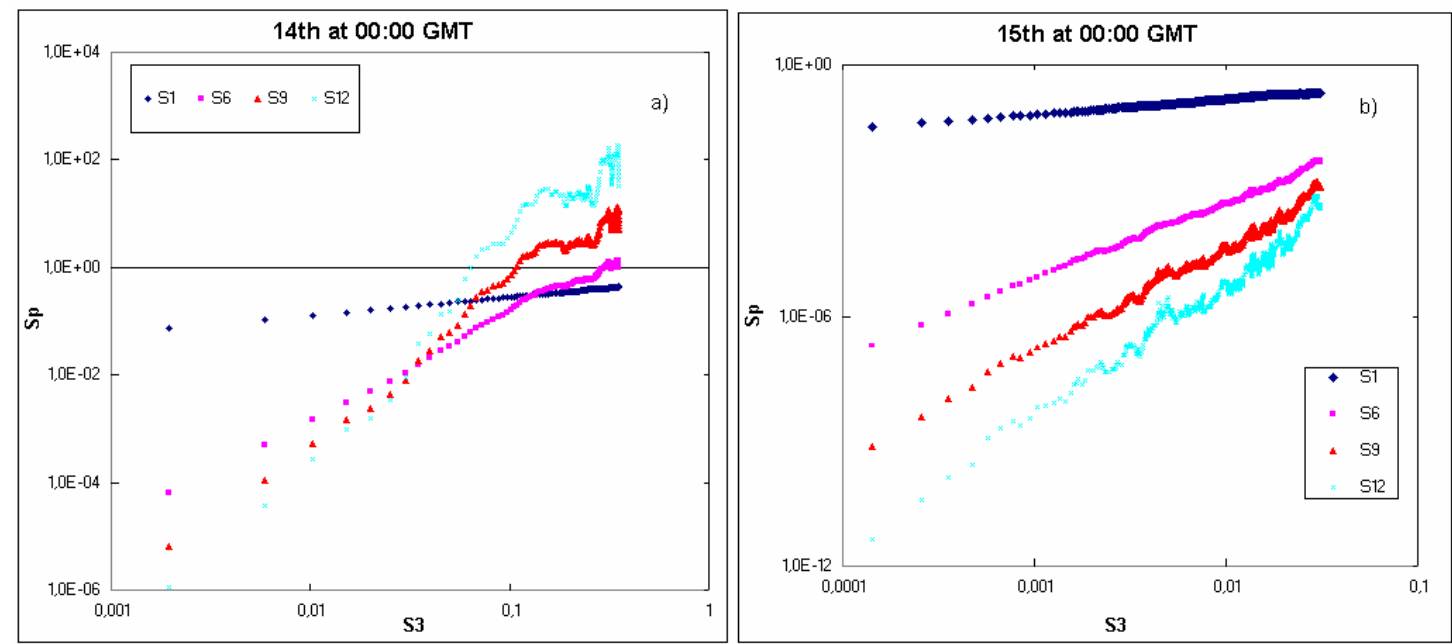

Fig. 10. Structure functions of several orders (1, 6,9 and 12) versus structure function of order 3 for the range below integral scale: (a) for the 14th at 00:00 GMT; (b) for the 15th at 00:00 GMT.
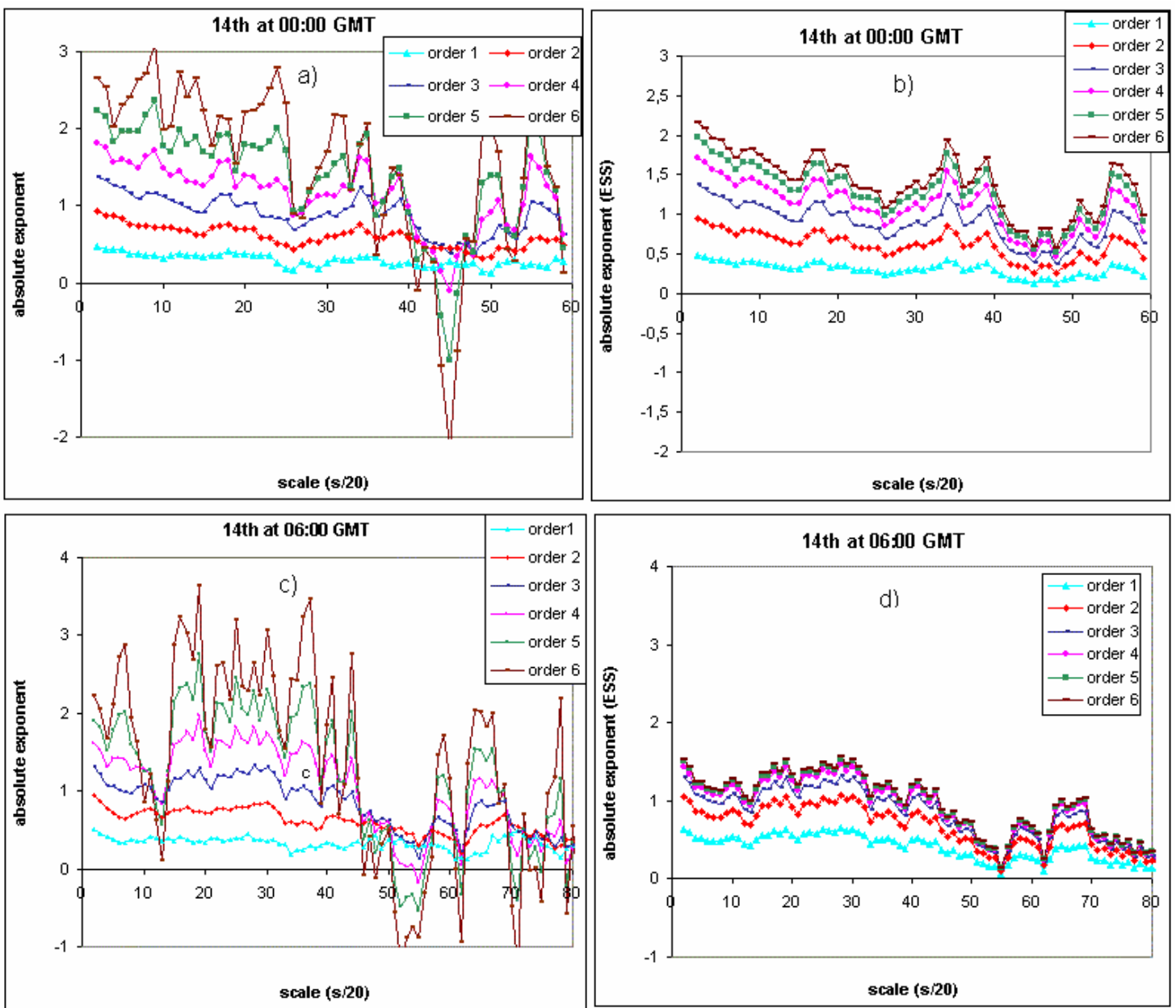

Fig. 11. Temporal evolution of absolute exponents (left) and absolute exponents re-evaluated by the ESS method (right) for the first 6 structure functions for the different situations: (a) and (b): 14th_00:00 GMT; (c) and (d): 14th_06:00 GMT. 


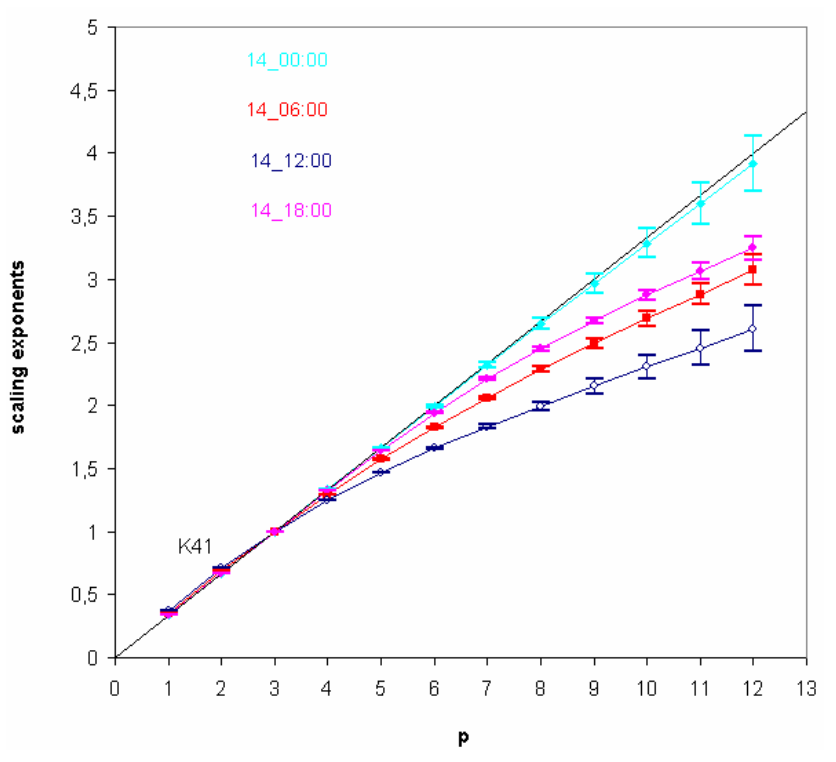

Fig. 12. Scaling exponents (ESS method) versus order of the structure function for different situations.

\section{Determination of inertial range in the Kolmogorov sense}

Table 1 shows the integral scales following the method of Van Fossen and Ching (1997) that reflects the upper scale of the inertial ranges corresponding to the different situations.

The empirical Kolmogorov 4/5 law establishes a relationship (for an incompressible flow in local equilibrium) between the third order structure function and the spatial scale:

$S_{3}(l)=-\frac{4}{5}\langle\varepsilon\rangle l$

This is a non-trivial result derived from Navier-Stokes equations. The interval that verifies this law (Eyink, 2003) is conventionally considered as the inertial range. In this case, the scaling exponents $\zeta_{p}$ are constant along the whole interval, indicating not only homogeneity and isotropy but also locality of the cascade process. Gotoh (2001) confirmed that this law holds using DNS at high Reynolds numbers.

Table 2 shows a series of possible inertial ranges for the situations under study. They have been obtained assuming a slope equal to 1 in Fig. 8, which relates the logarithm of the structure function of order 3 with the logarithm of the scale. We see that there are situations, such as 15 September at 00:00 GMT and at 06:00 GMT, in which it is not possible to obtain this interval. The slopes for other orders $p$ represent the scaling exponents $\zeta_{p}$.

The estimators of these slopes, $b^{*}$, will be affected by the error produced when the points are fitted to a straight line by the least squares method. Thus, setting a certain value $\gamma$ for the confidence interval for the slope $b$, it is possible to calculate the error interval for $b^{*}$. Comparing the relationship between $\zeta_{p}$ and $p$ with $p / 3$ (values for the exponents $\zeta_{p}$, foreseen by the K41 theory), the degree of intermittency may be established (Fig. 9, where a 99\% confidence interval has been chosen). The greater the deviation from $p / 3$, the greater the degree of intermittency in the cascade. According to this, the situations, within their corresponding inertial ranges of lesser to greater intermittency, are the following: on the 14th at 00:00 GMT, at 18:00 GMT, at 06:00 GMT and, with a slightly greater difference, at 12:00 GMT. This result agrees well with the conclusions that may be drawn from the previous figures, although these are not restricted to the inertial range evaluated in the Kolmogorov sense.

\section{Inertial range determination by the Extended Self- Similarity method (ESS)}

According to this method (Benzi et al., 1993), the inertial range is the interval where a potential relationship between the structure function and the structure function of order 3 is fulfilled:

$S_{p} \alpha S_{3} \overline{\zeta_{p}}$

The exponents $\overline{\zeta_{p}}$ are called relative exponents and are constant for this inertial range. Their values are:

$\overline{\zeta_{p}}=\zeta_{p} / \zeta_{3}$

Unlike the previous method, this one is not limited to homogeneity intervals. Therefore, the scaling exponent, usually known as the absolute exponent $\left(\zeta_{p}\right)$, is not now constant throughout the inertial range.

Using structure functions up to degree 12 , we have checked that Eq. (11) is verified for the ranges below integral scale (Table 1), with a determination coefficient of at least $R^{2}>0.85$ for all cases (Fig. 10).

The ESS procedure seems more general, since it eliminates the requirement for the third order structure functions to be unity for the whole range. This behaviour is typical of stable strongly non-homogeneous flows, such as those on 15 September at 00:00 GMT and 06:00 GMT.

An estimate of absolute exponents for each scale was made, multiplying the corresponding constant relative exponent by the absolute exponent of order 3. Figure 11a and c show a first approximation to the absolute exponents for each scale: the value of the absolute scaling exponents is the slope of the log. of the structure functions against the $\log$. of the scales (the slopes are obtained for two consecutive points). In the segments where the absolute exponents decrease, the relative exponents are almost constant (Mahjoub, 2000; Mahjoub et al., 1998, 2000, 2001). If these previous absolute exponents of order 3 are multiplied by the relative ones (corresponding to slopes of the log of the structure functions versus the $\log$ of structure functions of order 3 ), reevaluated absolute exponents are obtained (Fig. $11 \mathrm{~b}$ and d). In this figure, a similar shape is observed among all exponents for the different orders. 

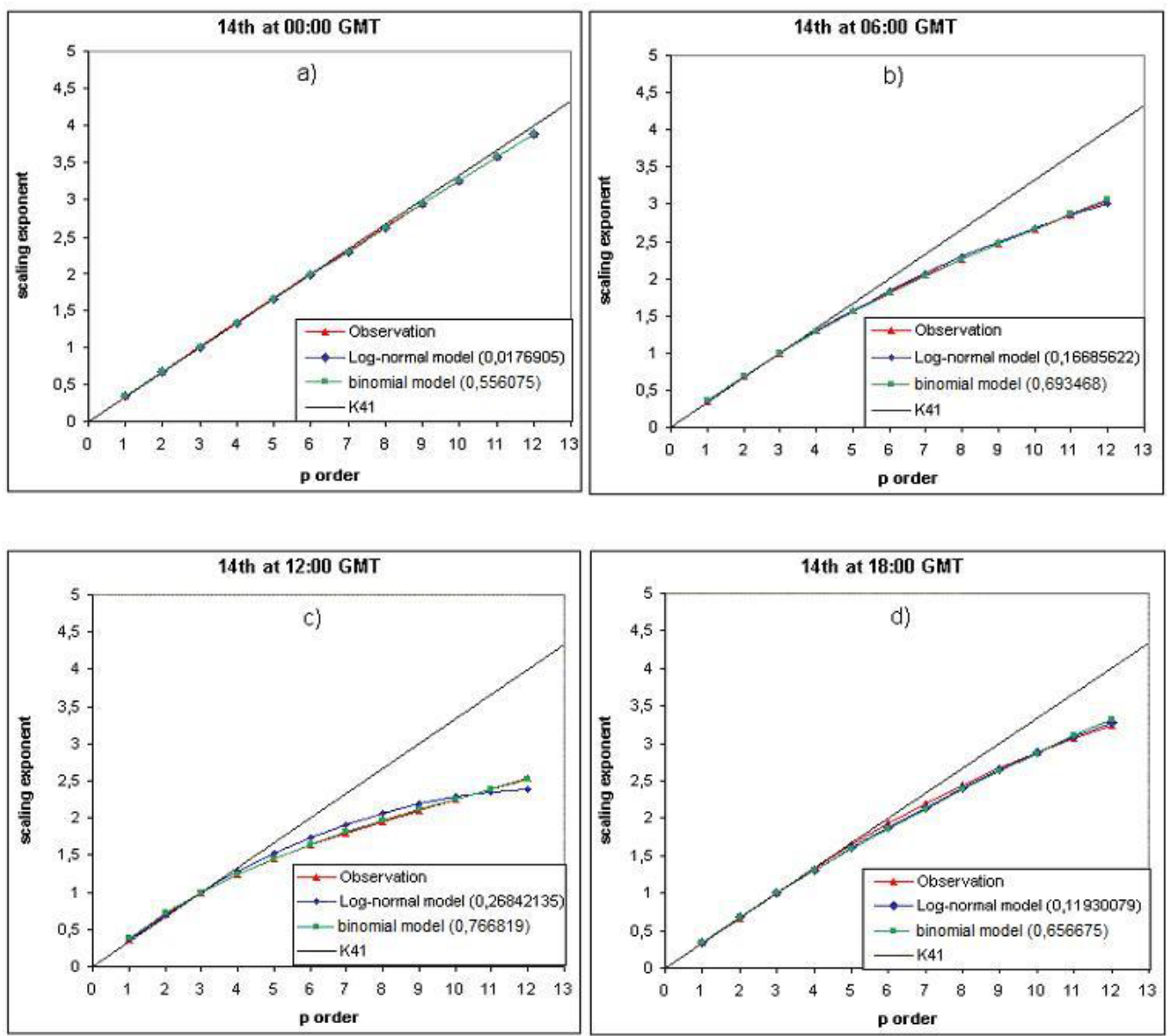

Fig. 13. Scaling exponents versus order of the structure function obtained by different methods: (a) 14th_00:00 GMT; (b) 14th_06:00 GMT; (c) 14th_12:00 GMT; (d) 14th_18:00 GMT.

In the subranges where homogeneity holds, such as those previously evaluated, we may use the relative exponents obtained, which are then equal to the absolutes, to determine the intermittency (Fig. 12). The results are practically identical to those obtained with the previous method, although the error bars are shorter, giving greater reliability to the results.

\section{Fits from theoretical models}

There are several models which seek to explain the profile that scaling exponents adopt when there is intermittency (Frisch, 1995). These models are characterized by one or more parameters whose values show the degree of intermittency.

For example, the binomial model (Meneveau and Sreenivasan, 1987): in the absence of intermittency the value of the intermittency parameter is 0.5 ; when intermittency increases, it also increases. The relationship between the parameter $(m)$ and the $p$ order scaling exponents is: $\zeta_{p}=1-\log _{2}\left(m^{p / 3}+(1-m)^{p / 3}\right)$

In the case of log-normal model (Kolmogorov, 1962), the intermittency parameter is $\mu$ (in the absence of intermittency, its value is 0 ; when intermittency increases, its value increases), being the relation between $\mu$ and scaling exponents:

$\zeta_{p}=\frac{p}{3}+\frac{\mu p}{18}(3-p)$

Using a least-squares fit, the parameters able to characterize intermittency according to the model (Fig. 13) have been estimated. We can see that the values of these parameters increase (from 0.5 for the binomial model and from 0 , in the case of the log-normal model) as the degree of intermittency is greater, in accordance with the results shown above. 


\section{Summary and conclusions}

We have performed a thorough analysis of the PDFs of the horizontal velocity differences for different atmospheric boundary layer situations and for different scales, and it has been seen the evolution of flatness with the scale, as well as that between structure functions and the degree of stratification.

In order to quantify these relationships, we adopted Kolmogorov's scaling to calculate the subrange where scaling exponents are constant. At the same time, using ESS we were able to recalculate the exponents (relative and absolute) for the same inertial subrange.

We have confirmed the applicability of the method, even if the resolution of the measurements only resolves the macroturbulence characteristics between the integral length scale and the Taylor microscale, but we do not claim that the turbulence is (necessarily) under local equilibrium.

Finally, using least-squares fits, the values of the parameters able to characterize the intermittency for the different stabilities, according to two different turbulence models (the binomial and the log-normal models) have been found.

We therefore highlight the following conclusions:

- The relationship existing between the structure functions and stratification shows that as stability increases the structure functions decrease.

- The variation of flatness $(F)$ with scale shows (Fig. 4) that the most stable (15th 06:00 GMT) and unstable situations (14th 12:00 GMT) have the highest values of $F$, but it is very interesting to note that for stably stratified flows, this happens at large scales.

- The overall results show that for convective, unstable turbulence intermittency $(\mu)$ increases. On the other hand, neutral conditions exhibit low intermittency.

- The determination of an inertial range in the Kolmogorov 1941 sense implies the identification of a homogeneity interval, which, in situations of high stability, is not always possible (15 September at 00:00 and 06:00 GMT).

- Absolute exponents have been shown, and no clear relationship between the degree of intermittency and the type of stratification has been established. However, the relationship between three ways of describing the intermittency has been pointed out: the evolution of flatness with the scale, the evolution of PDFs with the scale and the values of the absolute scaling exponents.

- Re-evaluating absolute exponents using ESS for the different orders, a more similar shape is observed among all exponents. Moreover, these re-evaluated exponents show a smoother shape than the initial exponents because of the compensating effect described in Mahjoub et al. (1998), and intermittency evaluated shows a higher degree of accuracy.

- The ESS method is applicable over the whole of the inertial subrange; but only within the subranges calculated assuming homogeneity and Kolmogorov scaling it is possible to compare intermittency for different situations. Otherwise we would need different intermittency parameters for each range of scales.

For strongly non-homogeneous flows, new types of intermittency parameters are needed that take into account the nonhomogeneity and the non-locality of the turbulent cascades.

Acknowledgements. This research has been funded by the Spanish Ministry of Education and Science (projects ESP2005-07551, CGL 2004-03109 and CGL 2006-12474-C03-03). The IV PRICIT program (supported by CM and UCM) has also partially financed this work through the Research Group "Micrometeorology and Climate Variability" (No. 910437). We thank the referees for their suggestions.

Edited by: A. Baas

Reviewed by: two anonymous referees

\section{References}

Angheluta, L., Benzi, R., Biferale, L., Procaccia, I., and Toschi, F.: Anomalous scaling exponents in nonlinear models of turbulence, Phys. Rev. Lett., 97, 1-4, 2006.

Anselmet, F., Gagne, Y., Hopfinger, E. J., and Antonia, R. A.: High-order velocity structure functions in turbulent shear flows, J. Fluid Mech., 140, 63-89, 1984.

Balbastro, G. C., Sonzogni, V. E., Franck, G., and Storti, M.: Acción del viento sobre cubiertas abovedadas aisladas: simulación numérica, Mecánica Computacional Vol. XXIII, Bariloche, Argentina, November 2004.

Batchelor, G. K. and Townsend, A. A.: The nature of turbulent motion at large wave numbers, Proc. Royal Soc. London A., 199 238-255, 1949.

Benzi, R., Ciliberto, S., Tripiccione, R., Baudet, C., Massaioli, F., and Succi, S.: Extended self-similarity in turbulent flows, Physical Review E., 48, R32, 1993.

Biferale, L.: Probability distribution functions in turbulent flows and shell models, Phys. Fluids A., 5, 428-435, 1993.

Biferale, L., Bodenschatz, E., Cencini, M., Lanotte, A. S., Ouellette, N. T., Toschi, F., and Xu, H.: Lagrangian structure functions in turbulence: A quantitative comparison between experiment and direct numerical simulation, Phys. Fluids, 20, 065103 , doi:10.1063/1.2930672, 2008.

Böttcher, F., Barth, S., and Peinke, J.: Small and large scale fluctuations in atmospheric wind speeds, Stochastic environmental research and risk assessment, 21, 299-308, 2007.

Chevillard, L., Castaing, B., and Lévêque, E.: On the rapid increase of intermittency in the near-dissipation range of fully developed turbulence, Eur. Phys. J. B., 45, 561-567, 2005.

Chevillard, L., Castaing, B., Lévêque, E., and Arneodo, A.: Unified multifractal description of velocity increments statistics in 
turbulence: intermittency and skewness, Physica D.: Non linear Phenomena, 218, 77-82, 2006.

Cuxart, J., Yagüe, C., Morales, G., Terradellas, E., Orbe, J., Calvo, J., Fernández, A., Soler, M. R., Infante, C., Buenestado, P., Espinalt, A., Joergensen, H. E., Rees, J. M., Vilá, J., Redondo, J. M., Cantalapiedra, I. R., and Conangla, L.: Stable atmospheric boundary-layer experiment in Spain (SABLES98): a report, Bound.-Lay. Meteor., 96, 337-370, 2000.

Eyink, G. L.: Local 4/5-law and energy dissipation anomaly in turbulence, Nonlinearity, 16, 137-145, 2003.

Frisch, U.: Turbulence, Cambridge University Press, England, 296 pp., 1995.

Giles, M. J.: Anomalous scaling in homogeneous isotropic turbulence, J. Phisics A.: Mathematical and General, 34, 4389-4435, 2001.

Glickman, Todd S.: Glossary of Meteorology, Second Edition, American Meteorological Society, Boston, 2000.

Gotoh, T.: Turbulence research at large Reynolds numbers using high resolution DNS, RIKEN Review, 40, 3-6, 2001.

Grossmann, S. and Lohse, D.: Scale resolved intermittency in turbulence, Phys. Fluids, 6, 611-617, 1994.

Kolmogorov, A. N.: Dissipation of energy in locally isotropic turbulence, C. R. Acad. Sci. USSR, 32, 16-18, 1941.

Kolmogorov, A. N.: A refinement of previous hypotheses concerning the local structure of turbulence in a viscous incompressible fluid at high Reynolds number, J. Fluid Mech. 13, 82-85, 1962.

Landau, L. D. and Lifshitz, E. M.: Fluids Mechanics, Pergamon Press, Oxford, 1959.

$\mathrm{Li}$, Y. and Meneveau, C.: Origin of non-gaussian statistics in hydrodynamic turbulence, Phys. Rev. Lett. 95, 164502, 1-4, 2005.

$\mathrm{Li}$, Y. and Meneveau, C.: Intermittency trends and lagrangian evolution of non-gaussian statistics in turbulent flow and scalar transport, J. Fluid Mech., 558, 133-142, 2006.

Mahjoub, O. B., Redondo, J. M., and Babiano, A.: Structure functions in complex flows, Appl. Sci. Res., 59, 299-313, 1998.

Mahjoub, O. B.: Non-local dynamics and intermittency in nonhomogeneous flows, $\mathrm{PhD}$, Technical University of Catalonia (UPC), 139 pp., 2000.
Mahjoub, O. B., Redondo, J. M., and Babiano, A.: Self similarity and intermittency in a turbulent non-homogeneous wake, edited by: Dopazo, C., 783-786, CIMNE, 2000.

Mahjoub, O. B., Granata, T., and Redondo, J. M.: Scaling laws in geophysical flows, Phys. Chem. Earth (B), 26, 281-285, 2001.

Meneveau, C. and Sreenivasan, K. R.: Simple multifractal cascade model for fully develop turbulence, Phys. Rev. Lett., 59, 1424 1427, 1987.

Nieuwstadt, F. T. M.: The turbulent structure of the stable nocturnal boundary layer, J. Atmos. Sci., 41, 2202-2216, 1984.

Richardson, L. F.: Weather prediction by numerical process, Cambridge Universtity Press, England, 236 pp., 1922.

Redondo, J. M., Sánchez, M. A., and Cantalapiedra, I. R.: Turbulent mechanisms in stratified fluids, Dyn. Atmos. Oceans, 24, 107115, 1996.

Rodriguez, A., Sanchez-Arcilla, A., Redondo, J. M., and Mosso, C.: Macroturbulence measurements with electromagnetic and ultrasonic sensors: a comparison under high-turbulent flows, Experiments in Fluids 27, 31-42, 1999.

Schumacher, J., Derivative moments in stationary homogeneous shear turbulence, J. Fluid Mech., 441, 109-118, 2001.

Sorriso-Valvo, L., Carbone, V., Veltri, P., Politano, H., and Pouquet, A.: Non-gaussian probability distribution functions in two dimensional magnetohydrodynamic turbulence, Europhys. Lett., 51, 520-526, 2000.

Stull, R. B.: An Introduction to Boundary Layer Meteorology, Atmospheric Sciences Library, Kluwer Academic Publishers, 666 pp., 1988.

Tennekes, H. and Lumley, J. L.: A first course in turbulence, MIT Press, Cambridge, MA, 1994.

Van Fossen, G. J. and Ching, C. Y.: Measurements of the influence of integral length scale on stagnation region heat transfer, International journal of rotating machinery, 3, 117-132, 1997.

Willis, G. E., and Deardorff, J. W.: On the use of Taylor's translation hypothesis for diffusion in the mixed layer, Quart. J. Roy. Meteor. Soc., 102, 817-822, 1976.

Yagüe, C., Viana, S., Maqueda, G., and Redondo, J. M.: Influence of stability on the flux-profile relationships for wind speed, $\emptyset_{\mathrm{m}}$, and temperature, $\emptyset_{\mathrm{h}}$, for the stable atmospheric boundary layer, Nonlin. Processes Geophys., 13, 185-203, 2006, http://www.nonlin-processes-geophys.net/13/185/2006/. 